\title{
THE LANDAU DISTRIBUTION FOR CHARGED PARTICLES TRAVERSING THIN FILMS
}

\author{
M. MARUCHO \\ Department of Chemistry, \\ Univeristy of Houston, \\ Houston, Texas, 77204-5003, USA. \\ mmarucho@uh.edu \\ C.A. GARCIA CANAL* AND HUNER FANCHIOTTI** \\ Laboratorio de Física Teórica, Departamento de Física, Universidad Nacional de La Plata, \\ C.C. 67 - 1900 La Plata, Argentina. \\ *garcia@fisica.unlp.edu.ar, **huner@fisica.unlp.edu.ar
}

\begin{abstract}
The Landau distribution as well as its first and second momenta are well suited for describing the energy loss of charged particles traversing a thin layer of matter. At present, just rational approximations and asymptotic expressions for these functions were obtained. In this paper we present a direct calculation of the integral representation of these functions obtaining perturbative and nonperturvative solutions expressed in terms of fast convergent series. We also provide a simple numerical algorithm which allows to control speed and precision of the results. The testing runs have provided, in reasonable computing times, correct results up to 13-14 significant digits on the density and distribution functions and 9-10 on the first and second momenta. If necessary, this accuracy could be improved by adding more coefficients to the algorithm.
\end{abstract}




\section{INTRODUCTION}

It is well known that the Bethe-Bloch formula[1] describes the average energy loss of charged particles when travelling through matter, while the fluctuations of energy loss by ionization of a charged particle in a thin layer of matter was theoretically described by Landau[2]. This description ends with a universal asymmetric probability density function characterized by a narrow peak with a long tail for positive values. This tail towards positive values comes from the small number of individual collisions, each with a small probability of transferring comparatively large amounts of energy. An integral representation of the Landau probability density function reads

$$
\phi(x)=\frac{1}{\pi} \int_{0}^{\infty} y^{-y} \sin (\pi y) \exp (-x y) d y
$$

with $x>0$.

Other functions such as the corresponding distribution function $\Phi(0, x)$ and the first and second momenta $\Phi(1, x)$ and $\Phi(2, x)$ of the density function truncated on the right-hand tail defined through the general formula

$$
\Phi(n, x)\left[\frac{(n-1)(n-2)}{2}+\frac{n(3-n)}{2} \Phi(0, x)\right]=\int_{-\infty}^{x} c^{n} \phi(c) d c
$$

are also needed in order to fit a truncated Landau distribution $\Phi(0, x)$ to measurements or to simulated energy-loss data [2, 3].

It is clear that a direct calculation, by numerical integration, of expressions (10) and (2) besides of being of a very slow convergence could be used only to provide values for a subsequent construction of approximate formula and for testing purposes.

In the seventies some programs for the numerical computation of the Landau distribution were presented[4]. There, the main emphasis was put more on the speed of the calculation algorithms than in the precision obtained, that was in general not good enough. In the eighties a new package of programs []ㅡㄹ arouse. These programs improved the precision of the values obtained for (11) and (2) by using piecewise functions as rational approximations and considering their asymptotic behavior. One should mention also Ref. 6 were more accurate calculations of the Landau density were presented. For more recent articles see Ref. 7. In view of the still high computational time required, the precision for the distribution function was only up to 6-7 significant digits. At that time, this was thought to be enough for the 
applications in experimental physics but their use in other fields of application remained excluded.

For practical uses and under the standard prejudice that equation (11) cannot be rewritten into an analytic form, the following approximate expression was suggested[8]

$$
\phi(x) \simeq \exp \left[-\frac{1}{2}(x+\exp (-x))\right] / \sqrt{2 \pi} .
$$

This analytic approximation, known as the Moyal function, is in fact an extreme value distribution [9]. It provides values that are not entirely coincident with those coming from the original expression (1), in particular in connection with its maximum value and its asymptotic behavior. Nevertheless, being quite simple, it is widely used in different fields. See Ref. 10 for examples.

To overcome the aforementioned difficulties, new and refined approximations, combining speed and accuracy in the evaluation of these functions, are required to improve the agreement between measured and calculated energy loss distributions.

Under this motivation, we have carried out a direct calculation of the integral representation of the Landau density, the distribution function and the two first momenta of the density. We have obtained perturbative and nonperturvative solutions expressed in terms of fast convergent series. To achieve this, we have used analytical continuation properties and the Laplace transform of the Landau density. We have also developed a simple numerical algorithm which allows one to control speed and precision of the results.

In what follows we summarize the main steps for obtaining the explicit analytic expressions for the Landau probability density (10) and the momenta (21). An appendix with details of the calculation is annexed. In addition, a brief description of the algorithm which evaluates numerically these functions is presented. Finally, a numerical test together with a critical analysis of the results is provided. 


\section{NONPERTURBATIVE SOLUTIONS}

\section{A. Landau density}

For computational purposes, it is of interest to consider the following auxiliary integral

$$
Q(x, b)=\frac{1}{\pi} \int_{0}^{\infty} y^{-y} \exp [-y(x+b)] d y .
$$

Clearly, once its solution is known, it is necessary to perform the analytic continuation

$$
\phi(x)=\operatorname{Im}[Q(x, b=-i \pi)]
$$

to obtain the Landau density.

By using standard representations of the functions in the integrand of $Q(c, b)$, one can write

$$
Q(x, b)=\frac{1}{\pi} \sum_{k=0}^{\infty} \frac{(-)^{k}}{k !} \frac{\partial^{k}}{\partial a^{k}}\left[\frac{\Gamma(k+a+1)}{(x+b)^{k+a+1}}\right]_{a=0}
$$

where $\Gamma$ is the gamma function[11]. Using now the identity

$$
\frac{\partial^{k}}{\partial a^{k}}[F(a) G(a)]=\sum_{r=0}^{k}\left(\begin{array}{l}
k \\
r
\end{array}\right) \frac{\partial^{k-r}}{\partial a^{k-r}}[F(a)] \frac{\partial^{r}}{\partial a^{r}}[G(a)]
$$

and defining the coefficients

$$
A_{k r} \equiv\left[\frac{\partial^{k-r}}{\partial a^{k-r}} \Gamma(k+a+1)\right]_{a=0}
$$

one obtains

$$
Q(x, b)=\frac{1}{\pi} \sum_{k=0}^{\infty} \frac{(-)^{k}}{k !} \sum_{r=0}^{k}\left(\begin{array}{l}
k \\
r
\end{array}\right)\left[\frac{A_{k r}}{(x+b)^{k+1}}\right](-)^{r}[\ln (x+b)]^{r} .
$$

Finally, performing the analytic continuation (5), one obtains the exact analytic expression for the Landau density

$$
\phi(x)=\frac{1}{\pi} \sum_{k=0}^{\infty} \frac{(-)^{k}}{k !} \sum_{r=0}^{k}\left(\begin{array}{l}
k \\
r
\end{array}\right)(-)^{r} A_{k r} \operatorname{Im}\left[\frac{[\ln (x-i \pi)]^{r}}{(x-i \pi)^{k+1}}\right] .
$$


Notice that this expression can be analytically continued to $x \leq 0$ values. However, we obtain below a formal solution also for this case, since the numerical evaluation of eq.(7) for negative values of $x$ makes the series divergent.

\section{B. Landau distribution and momenta}

In order to compute the functions defined in Eq.(2) we use the Laplace transform of the Landau density, namely

$$
\int_{-\infty}^{+\infty} \exp (-p c) \phi(c) d c=p^{p} \quad ; \quad p \geq 0 .
$$

The derivative of this relation with respect to $p$, gives rise to a new and convenient expression for computing the Landau distribution and the two first momenta of the density as follows

$$
\begin{gathered}
\Phi(n, x)\left[\frac{(n-1)(n-2)}{2}+\frac{n(3-n)}{2} \Phi(0, x)\right]= \\
\lim _{p \rightarrow 0^{+}}\left[(-)^{n}\left[\frac{n(n-1)(n-3 / 2)}{p}+(1+\ln (p))^{n}\right] p^{p}\right. \\
\left.-\int_{x}^{+\infty} c^{n} e^{-p c} \phi(c) d c\right] .
\end{gathered}
$$

Replacing now the Landau density (7), into this last expression, we see that the calculation is reduced to the computation of

$$
\begin{gathered}
G(p, x, n) \equiv \frac{1}{\pi} \sum_{k=0}^{\infty} \frac{(-)^{k}}{k !} \sum_{r=0}^{k}\left(\begin{array}{c}
k \\
r
\end{array}\right)(-)^{r} A_{k r} \\
\lim _{p \rightarrow 0^{+}} \operatorname{Im}\left[\int_{x}^{+\infty} c^{n} e^{-p c} \frac{[\ln (c-i \pi)]^{r}}{(c-i \pi)^{k+1}} d c\right]
\end{gathered}
$$

that means the integrals

$$
B_{k r}^{n}(p, x)=\lim _{p \rightarrow 0^{+}} \operatorname{Im}\left[\int_{x}^{+\infty} c^{n} e^{-p c} \frac{[\ln (c-i \pi)]^{r}}{(c-i \pi)^{k+1}} d c\right]
$$

Once these integrals are evaluated, an analysis of their eventual divergences and the necessary cancellations are in order. 


\section{Landau distribution}

Let us solve first the case $n=0$, the Landau distribution function. Notice that in this case all the integrals in expression (10) are well defined and consequently the interchange between the limit operation and the integral is licit. In this way we obtain

$$
B_{00}^{0}(0, x)=\operatorname{Im}\left[\int_{x}^{+\infty} \frac{1}{(c-i \pi)} d c\right]=\pi[1 / 2-\arctan (x / \pi) / \pi]
$$

and

$$
B_{k r}^{0}(0, x)=\lim _{b \rightarrow \infty^{+}} \operatorname{Im}\left[\int_{\ln (x-i \pi)}^{\ln (b-i \pi)} u^{r} \exp (-k u) d u\right] .
$$

A further change of variables to $v=u k$, reduces the integral to an incomplete Gamma function[11]. Finally, after taking the limit, one obtains

$$
B_{k r}^{0}(0, x)=\frac{1}{k^{r+1}} \operatorname{Im}[\Gamma(r+1, k \ln (x-i \pi))] .
$$

Going back to equation (21) one gets

$$
G(0, x, 0)=\frac{1}{2}-\frac{1}{\pi} \arctan \left(\frac{x}{\pi}\right)+\frac{1}{\pi} \sum_{k=1}^{\infty} \frac{(-)^{k}}{k !} \sum_{r=0}^{k}\left(\begin{array}{l}
k \\
r
\end{array}\right)(-)^{r} A_{k r} B_{k r}^{0}(0, x)
$$

so that the Landau distribution results

$$
\begin{gathered}
\Phi(0, x)=\frac{1}{2}+\frac{1}{\pi} \arctan \left(\frac{x}{\pi}\right) \\
-\frac{1}{\pi} \sum_{k=1}^{\infty} \frac{(-)^{k}}{k !} \sum_{r=0}^{k}\left(\begin{array}{l}
k \\
r
\end{array}\right) \frac{(-)^{r} A_{k r}}{k^{r+1}} \operatorname{Im}[\Gamma(r+1, k \ln (x-i \pi))] .
\end{gathered}
$$

\section{Momenta}

Going now to the analysis of the momenta, we found that in the general expression there appear divergences that finally cancel out, but that have to be treated with care. We postpone to an Appendix some details of the lengthy calculation involved and present here the final results, both for the first and the second momenta. They read

$$
\Phi(1, x) \Phi(0, x)=-1+\gamma+\frac{1}{2} \ln \left(x^{2}+\pi^{2}\right)
$$




$$
\begin{gathered}
+\frac{1}{\pi} \sum_{r=0}^{1}(-)^{r} A_{1 r}[\pi R e[\Gamma(r+1, \ln (x-i \pi))]+ \\
\left.\frac{1}{r+1}\left[1-r+r \ln \left(x^{2}+\pi^{2}\right)\right][\pi / 2-\arctan (x / \pi)]\right]-\frac{1}{\pi} \operatorname{Im} \sum_{m=0}^{1}(i \pi)^{1-m} \\
\sum_{k=2}^{\infty} \frac{(-)^{k}}{k !} \sum_{r=0}^{k}\left(\begin{array}{l}
k \\
r
\end{array}\right) \frac{(-)^{r} A_{k r}}{(k-m)^{r+1}}[\Gamma(r+1,(k-m) \ln (x-i \pi))]
\end{gathered}
$$

and

$$
\begin{aligned}
& \Phi(2, x) \Phi(0, x)=1+x+\frac{\pi}{2}[\pi-2 \arctan (x / \pi)] \\
& +(1-\gamma)\left[\frac{x^{2}}{\left(x^{2}+\pi^{2}\right)}-1-2\left[\gamma+\frac{1}{2} \ln \left(x^{2}+\pi^{2}\right)\right]\right] \\
& -\frac{1}{\pi}\left[\frac{x^{2}\left[x\left[\arctan (x / \pi)-\frac{\pi}{2}\right]+\frac{\pi}{2} \ln \left(x^{2}+\pi^{2}\right)+\pi\right]}{x^{2}+\pi^{2}}-2 \pi+\gamma \pi+\gamma^{2} \pi+\frac{\pi^{3}}{6}\right] \\
& -\frac{1}{\pi}\left[-\frac{\pi}{4}\left[\ln \left(x^{2}+\pi^{2}\right)\right]^{2}-2 x\left[\arctan (x / \pi)-\frac{\pi}{2}\right]+\pi\left[\arctan (x / \pi)-\frac{\pi}{2}\right]^{2}\right] \\
& -\frac{1}{2 \pi} \sum_{r=0}^{2}\left(\begin{array}{l}
2 \\
r
\end{array}\right)(-)^{r} A_{2 r} \operatorname{Im}\left[-\frac{[\ln (x-i \pi)]^{r+1}}{r+1}\right. \\
& \left.+\sum_{m=0}^{1}\left(\begin{array}{c}
2 \\
m
\end{array}\right) \frac{(i \pi)^{2-m}}{(2-m)^{r+1}}[\Gamma(r+1,(2-m) \ln (x-i \pi))]\right] \\
& -\frac{1}{\pi} \operatorname{Im} \sum_{m=0}^{2}\left(\begin{array}{c}
2 \\
m
\end{array}\right)(i \pi)^{2-m} \sum_{k=3}^{\infty} \frac{(-)^{k}}{k !} \\
& \sum_{r=0}^{k}\left(\begin{array}{l}
k \\
r
\end{array}\right) \frac{(-)^{r} A_{k r}}{(k-m)^{r+1}}[\Gamma(r+1,(k-m) \ln (x-i \pi))]
\end{aligned}
$$

being $\gamma$ the Euler's constant[11]. Notice that the limit $x \rightarrow \infty$ indicates that these distributions, even if normalized to 1 , do not have well defined momenta. 


\section{PERTURBATIVE SOLUTIONS}

\section{A. Landau density}

For the sole purpose of presenting solutions in an as simple as possible way, we define two auxiliary functions $M_{j}(x)$ and $T_{i j l}(x)$. First

$$
M_{j}(x) \equiv \frac{1}{\pi} \sum_{n=0}^{\infty} \frac{(-x)^{n}}{n !} \sum_{k=1}^{\infty}(-)^{k} \exp (-x k) \sum_{p=0}^{\infty} \frac{D_{j n k p}}{(2 p+1) ! 4^{p}}
$$

where the coefficients $D_{j n k p}$ are given by

$$
D_{j n k p}=\left.\frac{\partial^{2 p}}{\partial u^{2 p}}(u+k)^{(-j-1-u-k)} u^{n} \sin (\pi u)\right|_{u=1 / 2} .
$$

Note that the expression (16]) is the pertubative solution of the integral

$$
M_{j}(x)=\frac{1}{\pi} \int_{1}^{\infty} y^{-y-j-1} \sin (\pi y) \exp (-x y) d y .
$$

This solution is simply obtained by dividing the integration range in consecutive unit segments and expanding afterwards the exponential and trigonometric functions in the integrand.

The other auxiliary function is given by

$$
T_{i j l}(x) \equiv \frac{(-1)^{j}}{\pi} \sum_{n=0}^{\infty} \frac{(-x)^{n}}{n !} t_{i j l n}
$$

being the coefficients $t_{i j l n}$ given by the following expression

$$
t_{i j l n}=\sum_{m=0}^{\infty} \frac{(-1)^{m} \pi^{2 m+l}}{(2 m+l) !}\left[1-\frac{l(l-1) / 2}{2 m+3}\right] \sum_{k=0}^{\infty} \frac{(k+j) !}{k !(k+n+i+2 m+l+1)^{k+j+1}} .
$$

The expression (18) is the perturbative solution of the integrals

$$
\frac{1}{\pi} \int_{0}^{1} y^{-y+i} \cos (\pi y) \exp (-x y) \ln ^{j}(y) d y, \frac{1}{\pi} \int_{0}^{1} y^{-y+i} \sin (\pi y) \exp (-x y) \ln ^{j}(y) d y
$$

and

$$
\frac{1}{\pi} \int_{0}^{1} y^{-y+i}\left[-\cos (\pi y)+\frac{\sin (\pi y)}{\pi y}\right] \exp (-x y) \ln ^{j}(y) d y
$$


for $l=0,1$ and 2 respectively, which is obtained by expanding the exponential $\exp (-y \ln (y))$, and the other functions appearing in the integrand, in powers of their arguments.

As a consequence, the Landau density is obtained by dividing the range of integration in expression (11) into $[0,1] \times[1, \infty]$. Thus, we get

$$
\phi(x)=T_{001}(x)+M_{-1}(x) .
$$

\section{B. Landau distribution and momenta}

The computation of these functions follows the steps done for the case of the nonperturbative regime. In this case, the integrals appearing in Eq.(8) are now computed from the following relationship

$$
\int_{x}^{+\infty} c^{n} e^{-p c} \phi(c) d c=\frac{1}{\pi} \sum_{j=0}^{n}\left(\begin{array}{c}
n \\
j
\end{array}\right) j ! x^{n-j} \int_{0}^{\infty} \frac{y^{-y}}{(p+y)^{j+1}} \sin (\pi y) \exp (-x y) d y .
$$

In the limit $p$ going to zero, Eq.(8) evaluated at $n=0$ becomes

$$
\Phi(0, x)=1-\frac{1}{\pi} \int_{0}^{\infty} \frac{y^{-y}}{(p+y)} \sin (\pi y) \exp (-x y) d y .
$$

Let us observe that this integral is well defined allowing us to evaluate it at $p=0$. In this way, we obtain the following expression for the Landau Distribution

$$
\Phi(0, x)=1-\frac{1}{\pi} \int_{0}^{\infty} y^{-y-1} \sin (\pi y) \exp (-x y) d y .
$$

Analogously, we get for $n=1$ and $n=2$

$$
\begin{gathered}
\Phi(1, x) \Phi(0, x)=-1-\ln (p)-\frac{x}{\pi} \int_{0}^{\infty} y^{-y-1} \sin (\pi y) \exp (-x y) d y \\
-\frac{1}{\pi} \int_{0}^{\infty} \frac{y^{-y}}{(p+y)^{2}} \sin (\pi y) \exp (-x y) d y
\end{gathered}
$$

and 


$$
\begin{aligned}
& \Phi(2, x) \Phi(0, x)=1+3 \ln (p)+\frac{1}{p}+\ln ^{2}(p) \\
& -\frac{1}{\pi} \int_{0}^{\infty} \frac{y^{-y}}{(p+y)^{3}} \sin (\pi y) \Gamma(3, x(y+p)) d y
\end{aligned}
$$

corresponding to the first and second momentum respectively.

We can conveniently rewrite these last two equations as

$$
\Phi(1, x) \Phi(0, x)=-1-\ln (p)-x[1-\Phi(0, x)]-\frac{1}{\pi} \int_{0}^{\infty} \frac{y^{-y}}{(p+y)^{2}} \sin (\pi y) \exp (-x y) d y
$$

and

$$
\begin{gathered}
\Phi(2, x) \Phi(0, x)=1+(3+2 x) \ln (p)+\frac{1}{p}+\ln ^{2}(p)+2 x+x^{2}[1-\Phi(0, x)] \\
\quad+2 x \Phi(1, x) \Phi(0, x)-\frac{2}{\pi} \int_{0}^{\infty} \frac{y^{-y}}{(p+y)^{3}} \sin (\pi y) \exp (-x(y+p)) d y
\end{gathered}
$$

By simplicity we also divide the range of integration of the integrals present in (22), (25) and (26) as $[0,1] \times[1, \infty]$ such that we can easily evaluate them in terms of the two auxiliary functions previously defined. In particular, all the integrals defined in $[1, \infty]$ are well defined and consequently they can be evaluated at $p=0$ before being computed. The divergent behaviour in the parameter $p$ of the integrals defined in $[0,1]$ is extracted by integrating them by parts. Then, the remaining finite parts are also expressed in terms of the auxiliary functions.

In the case $n=0$ the integral defined in $[0,1]$ is well defined and we do not need to integrate it by parts. Consequently, Eq. (22) provides directly the final expression for the Landau Distribution

$$
\Phi(0, x)=1-M_{0}(x)-T_{-101}(x)
$$

which for the first and second momenta one has

$$
\begin{aligned}
\Phi(1, x)= & -\frac{x[1-\Phi(0, x)]+M_{1}(x)}{\Phi(0, x)} \\
& -\frac{T_{-121}(x)+(1+x) T_{-111}(x)+\pi T_{-112}(x)}{\Phi(0, x)}
\end{aligned}
$$


and

$$
\begin{aligned}
\Phi(2, x)= & \frac{1+3 x+x^{2}[1-\Phi(0, x)]+2 x \Phi(1, x) \Phi(0, x)}{\Phi(0, x)} \\
& -\frac{\exp (-x)\left[1-\pi^{2} / 3\right]-2 M_{2}(x)-I(x)}{\Phi(0, x)}
\end{aligned}
$$

respectively, where $I(x)$ is given by the expression

$$
\begin{aligned}
I(x)= & -\pi^{2}\left[T_{021}(x)+2(1+x) T_{011}(x)-\frac{\pi^{2}}{3} T_{001}(x)\right] \\
& -\pi T_{-112}(x)+(1+x) T_{-111}(x)-\pi T_{-101}(x) \\
& -\pi\left\{T_{030}(x)+3(1+x) T_{020}(x)-\frac{\pi^{2}}{3}(1+x) T_{000}(x)\right. \\
& \left.+\left[2(1+x)^{2}-\frac{\pi^{2}}{3}\right] T_{010}(x)\right\} .
\end{aligned}
$$

\section{ALGORITHM}

The nonperturbative expressions (7), (13), (14) and (15) are used for $x>3$, and the corresponding perturbative expressions (19), (27), (28) and (29) for $|x| \leq 3$. These expressions are the kernel of our algorithm.

In order to compute the nonperturbative expressions we have provided the algorithm with the following numerical coefficients

$$
B_{k r}=\frac{(-)^{k+r} A_{k r}}{r !(k-r) !}
$$

where $A_{k r}$ is given by (6). These coefficients were computed for $k>r$ by the following relationship

$$
A_{k r}=\left.\sum_{p=0}^{k-r-1}\left(\begin{array}{c}
k-r-1 \\
p
\end{array}\right) \frac{\partial^{p}}{\partial a^{p}} \Gamma(k+a+1) \Psi(k-r-1-p, k+a+1)\right|_{a=0}
$$

$\Psi(n, z)$ being the polygamma function[11] and $A_{k k}=\Gamma(k+1)$.

We computed these coefficients through the following numerical recursive algorithm 


$$
\text { for } j=0,(k-r) \rightarrow A(k, j)=\sum_{p=0}^{j-1}\left(\begin{array}{c}
j-1 \\
p
\end{array}\right) A(k, p) \Psi(j-1-p, k+1)
$$

where $A(k, 0) \equiv \Gamma(k+1)$. Consequently $A_{k r}=A(k, k-r)$. Replacing these coefficients into Eq.(31) we get the numbers $B_{k r}$. This algorithm was worked with MAPLE software.

Taking into account that our results provide excellent approximations, considering only a few terms of the corresponding series, we have just calculated the first 5050 coefficients corresponding to values of $k=0 \cdot 99$ and $r=0 \cdot k$

The computation for the functions $\Phi(0, x), \Phi(1, x)$ and $\Phi(2, x)$ involves, in addition, the evaluation of the Incomplete Gamma function with complex argument and integer order $\Gamma(n, z)$. It is accomplished by using the following expression[11]

$$
\Gamma(n, z)=(n-1) ! \exp (-z) \sum_{p=0}^{n-1} \frac{z^{p}}{p !} \quad n \geq 1 .
$$

On the other hand, the perturbative expressions are computed by providing the algorithm the following numerical coefficients

$$
D 2_{j n k p} \equiv \frac{D_{j n k p}}{(2 p+1) ! 4^{p}}
$$

where $D_{j n k p}$ is given by the Eq.(17). These coefficients were numerically computed for $0 \leq j \leq 3,0 \leq n \leq 26,1 \leq k \leq 20$ and $0 \leq p \leq 14$ using MAPLE software.

In addition we have defined auxiliary functions to compute the expressions (16), (18) and (30).

The data files containing the aforementioned numerical coefficients (31) and (33) can be found in the following link: www.fisica.unlp.edu.ar/download/landau.

\section{NUMERICAL RESULTS}

The test of our algorithm is based on the numerical integration of the expressions (11) and (2) using MAPLE software. We found that the algorithm allows to obtain results with a precision up to thirteen to fourteen significant digits for the Landau density and distribution and nine to ten for the first and second momenta. If necessary, higher accuracy can be obtained by adding more coefficients $B_{k r}$ and $D 2_{j n k p}$ into the algorithm. 
The set of points chosen to evaluate the aforementioned functions includes those values presented in Ref. 5 where the precision achieved certainly depends on the convergence of the expressions obtained. The corresponding numerical solutions for these functions are tabulated in the Tables 1,2,3 and 4. We also included in these Tables an estimation on the order of the series associated with our solutions by which a lower precision in the numerical results is obtained. Specifically, we have computed $|\Delta f(x)|=\left|f_{s+1}(x)-f_{s}(x)\right| \leq 10^{-\mathrm{d}}$, where $f(x)$ represents any of the following four functions; $\phi(x), \Phi(0, x), \Phi(1, x)$ and $\Phi(2, x)$, and " $s$ " represents the number of terms needed by the sum over the dummy variable $k$ (Eqs. (71), (13), (14) and (15)) or $n$ (Eqs.(16) and (18)) to obtain a precision of " $d$ " significant digits in the nonperturbative or perturbative regimes respectively.

Indeed, this information provides insight on how the convergence of our solutions depend on the point where the function is evaluated and on the required precision. In particular, we note that faster evaluations correspond to points localized closer to the origin or to the positive tails.

For getting some computing time estimation, we developed a partially optimized program written in Fortran 77 which is based on the above described algorithm. We found that the typical running time to compute, with a given accuracy, the four functions over the complete set of points is, in general, of the order of the fraction of a second in a common PC processor. For instances, the total number of CPU seconds that the process spent in user mode is around $0.205,0.197$ and 0.185 for $d$ equal to 12,9 and 6 respectively.

\section{SUMMARY AND DISCUSSION}

The Landau distribution as well as its first and second momenta are used to describe the energy loss of charged particles by ionization, particularly within the area of the high energy physics. At present, just rational approximations and asymptotic expressions for these functions were available. Numerical algorithms using these approximate solutions provide a fairly fast computation. In this paper we presented a direct calculation of the integral representation of these functions obtaining perturbative and nonperturvative solutions expressed in terms of fast convergent series. These solutions were obtained by using analytical continuation properties and the expression of the Laplace transform of the Landau density. They have been shown to be very flexible, allowing to combine accuracy and speed to adapt 
our approach to the computation of these functions to many fields of application. We remark that with only few terms of our series and a fast evaluation of them in all the range of its variables, we obtain excellent approximations. In fact, we found that our algorithm provides in reasonable computing times correct results up to 13-14 significant digits on the density and distribution functions and 9-10 on the first and second momenta. This is clearly a substantial numerical advantage over the existing programs mentioned above. If necessary, one can improve that precision by including further coefficients into the algorithm.

Future work involves the obtention of approximate analytical expressions for the single collision cross section and the electro dynamic properties of the detector medium[12]. These quantities, which can be computed in terms of the Landau distribution, could preserve the main features of our solutions. In particular they could be similarly expressed in terms of fast convergent series. This will surely improve not only its numerical evaluation but it will also allows to produce suitable fits of the truncated Landau distribution to measured or simulated energy-loss data. In fact, one should obtain an approximate analytical expression for the dielectric function (or equivalently the oscillator strength function) which is welldefined over the complete range of energy. This should remove the presence of non-physical high energy behaviour introduced in Ref. 12 by using a different approximation for the Landau distribution.

\section{Acknowledgments}

We thank Sergio Fanchiotti for stimulating discussion on the algorithm used. CAGC thanks J. Bernabeu and the Departamento de Física Teórica, Universidad de Valencia where part of this work was performed, for the warm hospitality extended to him. CAGC and HF acknowledge CONICET and Agencia Nacional de Promoción Científica for financial support.

\section{Appendix A: TECHNICAL DETAILS}

We start by analyzing the expression (10) $B_{k r}^{n}(p, x)$ where we cannot, a priori, interchange the operations of limit $p \rightarrow 0^{+}$and the integration. In those cases we are interested in the

obtention of their finite analytic contribution. Their divergent terms cancel with terms like $p^{p}\left[n(n-1)(n-3 / 2) / p+(1+\ln (p))^{n}\right]$ coming from Eq. (8) . Then we solve the other 
integrals where the interchange of operations simplifies the calculation.

In the case $n=1$ the divergent integral is just the first one which is given for $k=0$ and $r=0$, namely

$$
B_{00}^{1}(p, x)=\lim _{p \rightarrow 0^{+}} \operatorname{Im}\left[\int_{x}^{+\infty} c \exp (-p c) \frac{1}{(c-i \pi)} d c\right] .
$$

Integrating by parts and expanding for small $p$ we obtain

$$
B_{00}^{1}(p, x) \simeq \pi\left[\gamma+\frac{1}{2} \ln \left(x^{2}+\pi^{2}\right)+\ln (p)\right]+\mathcal{O}(p) .
$$

The other integrals corresponding to $(k \geq 1)$ can be rewritten, after a change of variables, as

$$
B_{k r}^{1}(0, x)=\operatorname{Im} \sum_{m=0}^{1}\left(\begin{array}{c}
1 \\
m
\end{array}\right)(i \pi)^{1-m} \lim _{b \rightarrow \infty^{+}}\left[\int_{\ln (x-i \pi)}^{\ln (b-i \pi)} u^{r} \exp [-u(k-m)] d u\right] .
$$

Taking into account that these integrals can be expressed in term of the incomplete Gamma function, we obtain:

- for $k>1$

$$
B_{k r}^{1}(0, x)=\operatorname{Im} \sum_{m=0}^{1}\left(\begin{array}{c}
1 \\
m
\end{array}\right)(i \pi)^{1-m} \frac{1}{(k-m)^{r+1}}[\Gamma(r+1,(k-m) \ln (x-i \pi))]
$$

- for $k=1$

$$
\begin{gathered}
B_{1 r}^{1}(0, x)=\pi \operatorname{Re}[\Gamma(r+1, \ln (x-i \pi))] \\
+\frac{1}{r+1}\left[1-r+r \ln \left(x^{2}+\pi^{2}\right)\right][\pi / 2-\arctan (x / \pi)]
\end{gathered}
$$

Replacing Eqs. (A1), (A2) and (A3) into Eq. (91) we check that the logarithmic divergent terms finally cancel and the remaining part of the integral provides the solution given by expression (14).

In the case $n=2$ the divergent integrals are for $k=0$ and $k=1$ and they read

$$
B_{00}^{2}(p, x)=\lim _{p \rightarrow 0^{+}} \operatorname{Im}\left[\int_{x}^{+\infty} c^{2} \exp (-p c) \frac{1}{(c-i \pi)} d c\right]
$$

and 


$$
B_{1 r}^{2}(p, x)=\lim _{p \rightarrow 0^{+}} \operatorname{Im}\left[\int_{x}^{+\infty} c^{2} \exp (-p c) \frac{[\ln (c-i \pi)]^{r}}{(c-i \pi)^{2}} d c\right]
$$

respectively. Integrating by parts and expanding for small values of $p$ we obtain

- for $k=0$ and $r=0$

$$
B_{00}^{2}(p, x) \simeq \pi\left[\frac{1}{p}-x-\frac{\pi}{2}[\pi-2 \arctan (x / \pi)]\right]+\mathcal{O}(p)
$$

- for $k=1$ and $r=0$

$$
B_{10}^{2}(p, x)=\pi\left[\frac{x^{2}}{\left(x^{2}+\pi^{2}\right)}-1-2\left[\gamma+\ln (p)+\frac{1}{2} \ln \left(x^{2}+\pi^{2}\right)\right]\right]+\mathcal{O}(p)
$$

- for $k=1$ and $r=1$

$$
\begin{gathered}
B_{11}^{2}(p, x)=\frac{x^{2}\left[x\left[\arctan (x / \pi)-\frac{\pi}{2}\right]+\frac{\pi}{2} \ln \left(x^{2}+\pi^{2}\right)+\pi\right]}{x^{2}+\pi^{2}}-2 \pi+\gamma \pi+\gamma^{2} \pi+\frac{\pi^{3}}{6} \\
-\frac{\pi}{4}\left[\ln \left(x^{2}+\pi^{2}\right)\right]^{2}-2 x\left[\arctan (x / \pi)-\frac{\pi}{2}\right]+\pi\left[\arctan (x / \pi)-\frac{\pi}{2}\right]^{2} \\
+\pi(1+2 \gamma) \ln (p)+\pi[\ln (p)]^{2}+\mathcal{O}(p)
\end{gathered}
$$

The others integrals $(k \geq 2)$ can be solved in the same way. The integrals can be written as

$$
\left.B_{k r}^{2}(0, x)=\operatorname{Im} \sum_{m=0}^{2}\left(\begin{array}{c}
2 \\
m
\end{array}\right)(i \pi)^{2-m} \begin{array}{l}
\lim \\
b \rightarrow \infty^{+}
\end{array} \int_{\ln (x-i \pi)}^{\ln (b-i \pi)} u^{r} \exp [-u(k-m)] d u\right]
$$

that allows one to obtain

- for $k>2$

$$
B_{k r}^{2}(0, x)=\operatorname{Im} \sum_{m=0}^{2}\left(\begin{array}{c}
2 \\
m
\end{array}\right)(i \pi)^{2-m} \frac{1}{(k-m)^{r+1}}[\Gamma(r+1,(k-m) \ln (x-i \pi))]
$$

- for $k=2$ 


$$
\begin{gathered}
B_{2 r}^{2}(0, x)=\operatorname{Im}\left[-\frac{1}{r+1}\left[[\ln (x-i \pi)]^{r+1}\right]\right. \\
\left.+\sum_{m=0}^{1}\left(\begin{array}{c}
2 \\
m
\end{array}\right)(i \pi)^{2-m} \frac{1}{(2-m)^{r+1}}[\Gamma(r+1,(2-m) \ln (x-i \pi))]\right]
\end{gathered}
$$

Replacing now Eqs.(A4), (A5), (A6), (A7) and (A8) into Eq.(9) we explicitly check that the divergent terms $1 / p, 3 \ln (p)$, and $\ln (p)^{2}$ finally cancel and the rest of the integral provides the solution given by the expression for the second moment.

[1] H. Bethe, Ann. Phys. 5, 325 (1930); H. Bethe, Z. Phys. 76, 293 (1932); F. Bloch, Ann. Phys. 16, 285 (1933); F. Bloch, Z. Phys. 81, 363 (1933).

[2] L. Landau, J. Phys. (USSR) 8, 201 (1944); in: L.D. Landau, Colleted Papers, ed. D. ter Haar (Pergamon, Press, Oxford, 1965), p. 417.

[3] S.M. Seltzer and M.J. Berger, Nucl. Sci. Series Rep. No. 39 (Nat. Acad. Sci. Washington, DC) (1964); W.W.M Allison and J.H. Cobb, Ann.Rev.Nucl.Sci 30, 253 (1980).

[4] B. Schorr, Comp. Phys. Comm. 7, 215 (1970).

[5] B. Schorr, Comp. Phys. Comm. 31, 84 (1984).

[6] H.D. Maccabee, D.G. Papworth, Phys. Lett. 30A, 241 (1969).

[7] CERNLB manual, G110, CERN program Library (1993); J.F. Ziegler, J. Appl. Phys./Rev. Appl. Phys. 85, 1249 (1999); see also http://www.star.bnl.gov/STAR /comp/simu/TpcRespSim/src/literature.html.

[8] J.E. Moyal, Phil. Mag. 46, 263 (1955). See also R.K. Bock et al., Formulae and Methods in Experimental Data evaluation with Emphasis on High Energy Physics, Vol 1, European Physical Society (1984).

[9] S. Kotz and S. Nadarajah, Extreme Value Distributions, Theory and Applications (Imperial College Press, 2000).

[10] C.O. Escobar, L.G. dos Santos and R.A. Vazquez, astro-ph/0202172. W. Adam et al., Nucl. Instr. and Meth. in Phys. Res.A 447, 244 (2000).

[11] M. Abramowitz and I. Stegun, Handbook of Mathematical Functions (Dover, New York, 1970); I.S. Gradshteyn and I.M. Ryzhik, Table of Integrals, Series and Products (Academic Press, New York, 2000). 
[12] O. Bärring, Nuclear Instrum. Meth.B 114, 1 (1996). 
Table 1

Numerical results for the Landau density. The integers given in columns 3-5 represent the number ofterms needed to achieve an accuracy of 12, 9 and 6 significant digits respectively.

\begin{tabular}{|c|c|c|c|c|}
\hline$x$ & $\phi(x)$ & $|\Delta \phi| \leq 10^{-12}$ & $|\Delta \phi| \leq 10^{-9}$ & $|\Delta \phi| \leq 10^{-6}$ \\
\hline-3 & $3.658377588806938 E-002$ & 20 & 16 & 12 \\
\hline-2.5 & $9.637807619860883 E-003$ & 18 & 15 & 11 \\
\hline-2 & $4.398547840053790 E-002$ & 17 & 14 & 10 \\
\hline-1.5 & 0.100550751729020 & 15 & 12 & 9 \\
\hline 0.4 & 0.168703915408766 & 10 & 8 & 6 \\
\hline 0.5 & 0.165232275482657 & 11 & 8 & 6 \\
\hline 1.5 & 0.124221094071471 & 16 & 13 & 9 \\
\hline 3 & $7.424765459960213 E-002$ & 21 & 18 & 14 \\
\hline 4 & $5.326865722328474 E-002$ & 29 & 17 & 8 \\
\hline 5 & $3.916341958128983 E-002$ & 21 & 13 & 7 \\
\hline 8 & $1.809028394181073 E-002$ & 10 & 9 & 7 \\
\hline 10 & $1.197648738878854 E-002$ & 11 & 9 & 6 \\
\hline 15 & $5.401336928010399 E-003$ & 10 & 8 & 6 \\
\hline 20 & $3.004979395692253 E-003$ & 10 & 8 & 6 \\
\hline 30 & $1.298671406775338 E-003$ & 9 & 7 & 5 \\
\hline 60 & $3.082734332814474 E-004$ & 8 & 6 & 4 \\
\hline 100 & $1.076112240078372 E-004$ & 7 & 6 & 4 \\
\hline 500 & $4.085712883475095 E-006$ & 5 & 4 & 2 \\
\hline 1000 & $1.012057179716136 E-006$ & 5 & 3 & 2 \\
\hline 10000 & $1.001659320662497 E-008$ & 3 & 2 & 0 \\
\hline
\end{tabular}


Table 2

Numerical results for the Landau distribution.

\begin{tabular}{|c|c|c|c|c|}
\hline$x$ & $\Phi(0, x)$ & $|\Delta \Phi| \leq 10^{-12}$ & $|\Delta \Phi| \leq 10^{-9}$ & $|\Delta \Phi| \leq 10^{-6}$ \\
\hline-3 & $-1.607879630341813 E-003$ & 20 & 17 & 13 \\
\hline-2.5 & $1.961867909885173 E-003$ & 19 & 16 & 12 \\
\hline-2 & $1.409435793315372 E-002$ & 17 & 14 & 11 \\
\hline-1.5 & $4.982427497490971 E-002$ & 15 & 13 & 9 \\
\hline 0.4 & 0.356581474467521 & 10 & 8 & 6 \\
\hline 0.5 & 0.373280562479680 & 11 & 9 & 6 \\
\hline 1.5 & 0.518343029194760 & 16 & 13 & 10 \\
\hline 3 & 0.664205997524218 & 21 & 18 & 14 \\
\hline 4 & 0.727271852143877 & 26 & 15 & 8 \\
\hline 5 & 0.773026779942834 & 18 & 12 & 7 \\
\hline 8 & 0.853459958739293 & 12 & 9 & 7 \\
\hline 10 & 0.882938865913564 & 11 & 9 & 7 \\
\hline 15 & 0.923293944585224 & 11 & 9 & 6 \\
\hline 20 & 0.943462646851257 & 10 & 8 & 6 \\
\hline 30 & 0.963250172910313 & 10 & 8 & 6 \\
\hline 60 & 0.982280577955328 & 9 & 7 & 5 \\
\hline 100 & 0.989571029163290 & 8 & 6 & 5 \\
\hline 500 & 0.997976630597665 & 6 & 5 & 3 \\
\hline 1000 & 0.998993481974151 & 6 & 4 & 3 \\
\hline 10000 & 0.999899912058608 & 4 & 3 & 1 \\
\hline
\end{tabular}


Table 3

Numerical results for the first momenta of the density.

\begin{tabular}{|c|c|c|c|}
\hline$x$ & $\Phi(1, x)$ & $|\Delta \Phi(1, x)| \leq 10^{-9}$ & $|\Delta \Phi(1, x)| \leq 10^{-6}$ \\
\hline-3 & -2.94418823883569 & 21 & 18 \\
\hline-2.5 & -2.67471836189293 & 19 & 16 \\
\hline-2 & -2.25828545384356 & 17 & 13 \\
\hline-1.5 & -1.86990165100088 & 14 & 11 \\
\hline 0.4 & -0.679860050176673 & 9 & 6 \\
\hline 0.5 & -0.629322356963745 & 9 & 7 \\
\hline 1.5 & -0.180019038237684 & 13 & 10 \\
\hline 3 & 0.339504262821524 & 18 & 14 \\
\hline 4 & 0.611170716736155 & 15 & 7 \\
\hline 5 & 0.839831585464024 & 12 & 7 \\
\hline 8 & 1.35514739591618 & 9 & 7 \\
\hline 10 & 1.60809603494204 & 9 & 7 \\
\hline 15 & 2.06972587987781 & 9 & 7 \\
\hline 20 & 2.39437506213547 & 8 & 6 \\
\hline 30 & 2.84447000017431 & 8 & 6 \\
\hline 60 & 3.59268530830209 & 7 & 5 \\
\hline 100 & 4.12998818980124 & 7 & 5 \\
\hline 500 & 5.77818474253184 & 5 & 4 \\
\hline 1000 & 6.47746787601202 & 5 & 3 \\
\hline 10000 & 8.78657692470135 & 4 & 3 \\
\hline
\end{tabular}


Table 4

Numerical results for the second momenta of the density.

\begin{tabular}{|c|c|c|c|}
\hline$x$ & $\Phi(2, x)$ & $|\Delta \Phi(2, x)| \leq 10^{-9}$ & $|\Delta \Phi(2, x)| \leq 10^{-6}$ \\
\hline-3 & 8.66852146374132 & 24 & 21 \\
\hline-2.5 & 7.17765680763846 & 22 & 19 \\
\hline-2 & 5.14686389834850 & 18 & 15 \\
\hline-1.5 & 3.58354569903627 & 16 & 13 \\
\hline 0.4 & 0.941718427356982 & 9 & 7 \\
\hline 0.5 & 0.908679035129438 & 10 & 7 \\
\hline 1.5 & 0.944186293458727 & 15 & 12 \\
\hline 3 & 1.82656174148077 & 21 & 9 \\
\hline 4 & 2.72090902630219 & 18 & 8 \\
\hline 5 & 3.74974971267330 & 13 & 8 \\
\hline 8 & 7.21517917106136 & 10 & 7 \\
\hline 10 & 9.64861303605968 & 10 & 7 \\
\hline 15 & 15.7892588337345 & 10 & 5 \\
\hline 20 & 21.8615020920748 & 9 & 8 \\
\hline 30 & 33.7144809006428 & 9 & 8 \\
\hline 60 & 67.7261171683676 & 8 & 8 \\
\hline 100 & 111.315504919124 & 8 & 7 \\
\hline 500 & 525.970930432499 & 7 & 7 \\
\hline 1000 & 1033.83849293122 & 6 & 7 \\
\hline 10000 & 10066.7727522736 & 5 & 7 \\
\hline
\end{tabular}

